\title{
EQUILIBRIUM IN A MARKET WITH INTERMEDIATION IS WALRASIAN
}

\author{
John Wooders*
}

\begin{abstract}
We show that a profit maximizing monopolistic intermediary may behave approximately like a Walrasian auctioneer setting bid and ask prices nearly equal to Walrasian equilibrium prices. In the model agents trade either through the intermediary or privately. Buyers (sellers) choosing to trade through the intermediary potentially trade immediately at the ask (bid) price, but sacrifice the spread as potential gains. Agents trading privately capture all of the gains to trade, but risk costly delay in finding a partner. We show that when the cost of delay is small, the intermediary sets bid and ask prices nearly equal to Walrasian equilibrium prices. As the cost of delay vanishes, the equilibrium bid and ask prices converge to the Walrasian equilibrium prices. If the possibility of trading through the intermediary is removed, and therefore all trade takes place in the private trading market, then prices are not close to Walrasian equilibrium prices even as the cost of delay vanishes.
\end{abstract}

Keywords: intermediation, Walrasian equilibrium, matching, bargaining.

* John Wooders, Departamento de Economía, Universidad Carlos III de Madrid and Department of Economics, University of Arizona, McClelland Hall, Tucson, Arizona, 85718. Tel: (602) 6216231, e-mail: jwooders@bpa.arizona.edu

I am grateful to the Spanish Ministry of Education for financial support. This paper is based on the essay "Intermediation with Inside Trading Opportunities" in my $\mathrm{Ph}$. D. dissertation. I am grateful to Larry Blume, David Easley, Robert Jarrow, Diego Moreno, and Mark Walker for their comments. 


\section{Introduction}

The notion of a benevolent auctioneer is sometimes used to explain the following paradoxical aspect of competitive equilibrium. In competitive economies each agent takes prices as given. But when all agents take prices as given, the process by which prices come to be equilibrium prices is left unexplained. The role of the auctioneer is to adjust prices until markets clear, thus resolving the paradox. (See Hahn [4] for a discussion of the auctioneer.) Our objective is to show that a self-interested monopolist intermediary may effectively play the role of a benevolent Walrasian auctioneer by setting nearly Walrasian bid and ask prices.

In our model agents have the option of trading through a monopolistic intermediary or trading privately in a random matching market. The intermediary strategically chooses bid and ask prices to maximize its own profits. Given the intermediary's bid and ask price, at each date buyers and sellers remaining in the market choose whether to attempt to trade with the intermediary or whether to attempt to trade privately. Agents entering the mediated market potentially trade immediately with the intermediary at the bid or ask price, but sacrifice the spread as potential gains to trade. Agents entering the private trading market capture all gains to trade once matched, but risk costly delay in finding a trading partner. In equilibrium each agent follows an optimal policy of entering the mediated and private trading market given (a) the bid and ask price, (b) the price negotiated in the private trading market, and (c) the entry policy of all other agents.

We show that when the costs of delay are small the intermediary sets bid and ask prices nearly equal to Walrasian equilibrium prices. The costs of delay arise from the discounting by agents of the utility from consumption in the future. We show that as the discount factor approaches one, the cost of delay therefore vanishing, the equilibrium bid and ask prices converge to the Walrasian equilibrium prices. In fact, for the economy under consideration intermediation is necessary for a Walrasian outcome. In the absence of intermediation all trade must take place in the private trading market and prices are not close to Walrasian prices, even as the cost of delay vanishes.

These results are obtained for an economy in which each agent has an interest in carrying out only one transaction. There are two goods, money and an indivisible good, at each of an infinite number of dates. A generation $t$ buyer who at date $\tau$ exchanges $p$ units of money for 
a unit of the indivisible good obtains utility $\delta^{\tau-t}(1-p)$, where $\delta$ is the discount factor. A generation $t^{\prime}$ seller who takes the other side of such an exchange obtains utility $\delta^{\tau-t^{\prime}} p$. The demographics are such that there is a greater measure of generation zero sellers than generation zero buyers, and for each generation $t$ thereafter there is an equal measure of sellers and buyers of that generation.

The equilibrium with intermediation is first compared to the equilibrium of a Walrasian trading procedure and then to the equilibrium of a trading procedure where there is only private trade. Walrasian equilibrium is defined to represent equilibrium in a situation where at each date there is a spot market for each good. The Walrasian trading procedure is interpreted as a centralized trading procedure; at each date agents can trade at the prevailing spot market prices without search. Formally, a Walrasian equilibrium is a sequence of spot market prices and an allocation such that the market for each good at each date clears and the allocation of each agent maximizes his utility given his budget. In Section 2 we show that the Walrasian equilibrium price of the indivisible good (in units of money) is zero at each date.

Section 3 describes the model of intermediation. In this model prices need not clear markets. Transaction prices in the mediated market are the bid and the ask price, while in the private trading market they are determined by Nash bargaining. ${ }^{1}$ In section 4 we show that in equilibrium, at each date the indivisible good is bought by the intermediary at a price of zero and sold at a price which is positive, but less than one. Thus, the intermediary and buyers capture all of the gains to trade. The difference in the prices is the "spread," which represents the profit to the intermediary of transacting a single unit of the indivisible good. As the discount factor approaches one, we show that in the limit the indivisible good is bought and sold at a price of zero at each date, and each buyer and seller obtains his Walrasian equilibrium payoff.

Section 5 discusses the situation when there is only private trading, in which case prices at each date are determined by bargaining. The private trading market is modeled as a random matching market and is therefore related to the models in Diamond [1], Gale [2], and Rubinstein and Wolinsky [8]. When there is only private trading, the equilibrium price of the indivisible good is greater than zero at each date. The price is positive, and the payoff of each agent different from his Walrasian equilibrium payoff, even in the limit as the discount factor approaches one.

\footnotetext{
${ }^{1}$ Using Nash rather than strategic bargaining simplifies the analysis and clarifies the intuition behind the results. Results similar to those reported here were obtained in Wooders [11] using strategic bargaining.
} 
In the context of strategic bargaining this result is well known from Rubinstein and Wolinsky [8].

For concreteness the gains to trade are taken to arise from exchange. The model of intermediation, therefore, is a model of intermediation in a goods market. Alternatively, one can take the gains as arising from the marriage of agents or as arising from the joint production of a divisible output. If the gains arise from marriage, then the intermediary is a matchmaker, matching boys to girls. If the gains arise from joint production, then the intermediary is an employment agency, matching workers to firms. In either case, the formalism of the model of intermediation is unchanged.

The intermediary in the present paper is a monopolist and is "large" in the sense that its choice of a bid and ask price influences the composition of the private trading market. Rubinstein and Wolinsky [9] consider a model of intermediation with many "small" intermediaries (or middlemen), where the activity of any one intermediary has no influence on market aggregates. They show that the distribution of the gains to trade is biased in favor of buyers when intermediaries take ownership of the good as opposed to when they trade on consignment. It is an open question whether the introduction of many small intermediaries of the kind in [9] makes the market outcome more nearly competitive.

\section{The Economy and Its Walrasian Equilibrium}

We consider an economy where each agent has an interest in carrying out only one transaction. Each buyer is concerned with the date and the price at which he obtains a unit of the indivisible good. Each seller is concerned with the date and the price at which he supplies a unit of the indivisible good. It is convenient to represent such an economy as an overlapping-generations economy with infinitely lived agents.

\section{The Economy}

The set of agents is denoted by $G$, where $G \subset \Re$. The Lebesgue measure on the line is denoted by $\mu$. Let $\left\{G_{B}, G_{S}\right\}$ be a Lebesgue measurable partition of $G$, where $G_{i}$ is the set of agents of type $i$. The indices $B$ and $S$ refer to "buyers" and "sellers," respectively. Time is indexed by $t \in\{0,1, \ldots\}$. Let $\left\{G^{0}, G^{1}, \ldots\right\}$ be another Lebesgue measurable partition of 
$G$, where $G^{t}$ is the set of agents born at date $t$. The set of generation $t$ agents of type $i$ is $G_{i}^{t}=G_{i} \cap G^{t}$. The demographics of the economy are such that there is a greater measure of generation zero sellers than generation zero buyers, and an equal measure of sellers and buyers at every generation thereafter. In particular, there exist numbers $\nu>0$ and $\Delta>0$ such that $\mu\left(G_{S}^{0}\right)=\nu+\Delta, \mu\left(G_{B}^{0}\right)=\nu$, and $\mu\left(G_{S}^{t}\right)=\mu\left(G_{B}^{t}\right)=\nu$ for each $t>0$. Therefore, by date $t$ a measure $\nu t+\Delta$ of sellers has been born while only a measure $\nu t$ of buyers has been born.

At each date there are two goods. Good 0 is divisible and plays the role of money, while good 1 is indivisible. An allocation for agent $h$ is a pair $\left(x_{h}, \tau_{h}\right)$ where $x_{h}=\left(x_{h}^{0}, x_{h}^{1}\right), x_{h}^{j}$ is the consumption of good $j$ by agent $h$, and $\tau_{h}$ is the date at which agent $h$ consumes. We assume for each agent $h$ that $x_{h}^{0} \in \Re$ (money holdings may be negative) and $x_{h}^{1} \in\{0,1\}$. Each agent may consume no earlier than the date at which he is born. Therefore, the consumption set of each agent $h \in G^{t}$ is

$$
X^{t}=\Re \times\{0,1\} \times\{t, t+1, \ldots\},
$$

where $\left(x_{h}^{0}, x_{h}^{1}, \tau_{h}\right) \in X^{t}$ denotes the allocation of agent $h$. An allocation is a pair of functions $(x, \tau)$, each mapping $G$ to $\cup_{t=0}^{\infty} X^{t}=X^{0}$, such that for each $t$ we have:

(i) $(x, \tau): G^{t} \rightarrow X^{t}$

(ii) For each $j \in\{0,1\}: x^{j}$ is integrable on $\left\{h: \tau_{h}=t\right\}$.

Condition $(i)$ is that the allocation of each agent is in his consumption set. Condition (ii) insures that it is meaningful to speak of the measure of good $j$ consumed by agents trading at date $t$.

Agent $h$ 's utility from the allocation $\left(x_{h}, \tau_{h}\right)$ is the utility that he obtains from consumption, $u_{h}\left(x_{h}^{0}, x_{h}^{1}\right)$, discounted from the date he consumes, $\tau_{h}$, back to the date he was born. In particular, from the allocation $\left(x_{h}, \tau_{h}\right)$ agent $h \in G^{t}$ obtains utility

$$
\delta^{\tau_{h}-t} u_{h}\left(x_{h}^{0}, x_{h}^{1}\right)
$$

where $\delta$, the discount factor, is less than one. The endowment of each buyer $h \in G_{B}^{t}$ is $\left(\omega_{h}, t\right)$ where $\omega_{h}=(0,0)$. Although the income of each buyer is zero, each buyer has a non-trivial budget set since consumption of money may be negative. Each seller is endowed with a unit of the indivisible good at the date he is born. In other words, the endowment of each seller $h \in G_{S}^{t}$ is $\left(\omega_{h}, t\right)$ where $\omega_{h}=(0,1)$. 
We assume for each buyer $h \in G_{B}$ that

$$
u_{h}\left(x_{h}^{0}, x_{h}^{1}\right)= \begin{cases}x_{h}^{0}+1 & \text { if } x_{h}^{1}=1 \\ x_{h}^{0} & \text { if } x_{h}^{1}=0\end{cases}
$$

and for each seller $h \in G_{S}$ that $u_{h}\left(x_{h}^{0}, x_{h}^{1}\right)=x_{h}^{0}$. Given these preferences and endowments, a generation $t$ buyer whose allocation is obtained by an exchange at date $s \geq t$ of $p_{s}$ units of money for a unit of the indivisible good has utility $\delta^{\boldsymbol{s}-t}\left(1-p_{s}\right)$. A generation $t^{\prime}$ seller whose allocation is obtained by taking the other side of such an exchange has utility $\delta^{8-t^{\prime}} p_{s}$. Thus, a buyer and seller have an (undiscounted) unit gain to trade.

\section{Walrasian equilibrium (in spot markets)}

Walrasian equilibrium as defined here represents equilibrium in a situation where trade is centralized; at each date, agents may trade at the prevailing prices without search. We take the Walrasian trading procedure to be one where $(i)$ at each date there is only a spot market for each good, and (ii) each agent concentrates his purchases and sales on spot markets at one date. This setup parallels the one in our model of the intermediated market and our model of the market with only private trade. In both of these models, $(i)$ exchanges at date $t$ involve only date $t$ money and date $t$ indivisible good, and (ii) each buyer and seller participates in only one exchange. Defining the Walrasian trading procedure in this fashion isolates the differing degrees of centralized trade under the different trading procedures (rather than, say, the presence or absence of futures markets) as the source of the differences in the market outcomes.

Since there is only a spot market for each good at each date, the price of money can be normalized to one at each date. ${ }^{2}$ Let $p_{s}$ denote the price of a unit of the indivisible good at date $s$ in terms of units of money at date $s$. Let $\mathbf{p}=\left(p_{0}, p_{1}, \ldots\right)$.

\footnotetext{
${ }^{2}$ We show that the price of money can be normalized to one at each date. For each $j \in\{0,1\}$ and each $t \geq 0$, let $p^{s, j}$ denote the price of good $j$ at date $s$. If agent $h$ concentrates his purchases and sales on spot markets at date $s$, his budget constraint at date $s$ is $p^{s, 0} x_{h}^{0}+p^{s, 1} x_{h}^{1} \leq p^{s, 0} \omega_{h}^{0}+p^{\infty, 1} \omega_{h}^{1}$. The problem of agent $h \in G^{t}$ is to choose a date $\tau_{h}$ at which to trade, and a feasible consumption $x_{h}$ given his budget constraint at $\tau_{h}$. Given prices $\left(p^{0,0}, p^{0,1}, \ldots, p^{s, 0}, p^{s, 1}, \ldots\right)$, agent $h \in G^{t}$ solves

$$
\max _{\left(x_{h}, \tau_{h}\right) \in X^{i}} \delta^{\tau_{h}-t} u_{h}\left(x_{h}\right) \text { s.t. } \cdot p^{\tau_{h}, 0} x_{h}^{0}+p^{\tau_{h}, 1} x_{h}^{1} \leq p^{\tau_{h}, 0} \omega_{h}^{0}+p^{\tau_{h}, 1} \omega_{h}^{1} .
$$

If $\left(x_{h}, \tau_{h}\right)$ is a solution to this problem, then it is also a solution given prices $\left(1, \frac{p^{0,2}}{p^{0,0}}, \ldots, 1, \frac{p^{0,1}}{p^{0,0}}, \ldots\right)$. Denote by $p_{s}=\frac{p^{0,2}}{p^{s, 0}}$ the price of date $s$ indivisible good in terms of date $s$ money.
} 
Given an allocation $(x, \tau)$, the set of agents trading at date $s$ is $\left\{h \in G: \tau_{h}=s\right\}$. When each agent concentrates his purchases and sales at one date, aggregate excess demand for commodity $j$ at date $s$ is

$$
\int_{\left\{h \in G \mid \tau_{h}=s\right\}}\left(x_{h}^{j}-\omega_{h}^{j}\right) d h .
$$

A Walrasian equilibrium is a price sequence and an allocation for each agent such that (i) the market for each good at each date clears, and (ii) each agent's allocation maximizes his utility given his budget. ${ }^{3}$

Definition: A Walrasian equilibrium is a pair $(\mathbf{p},(x, \tau))$ satisfying for all $t \geq 0$ :

(i) For each $j \in\{0,1\}$ :

$$
\int_{\left\{h \in G \mid \tau_{h}=t\right\}}\left(x_{h}^{j}-\omega_{h}^{j}\right) d h \leq 0
$$

(ii) For each $h \in G^{t}$ :

$$
\left(x_{h}, \tau_{h}\right) \in \arg \max _{\left(x_{h}, \tau_{h}\right) \in X^{t}} \delta^{\tau_{h}-t} u_{h}\left(x_{h}^{0}, x_{h}^{1}\right) \text { s.t. } x_{h}^{0}+p_{\tau} x_{h}^{1} \leq \omega_{h}^{0}+p_{\tau} \omega_{h}^{1} .
$$

Although there is an infinite measure of each type of agent in the economy, the integration in $(i)$ is over a set of agents of finite measure since the set of agents who can feasibly trade at date $t$ (i.e. the set of agents born at or prior to date $t$ ) has measure $2 \nu t+\Delta<\infty$. We say that $\mathbf{p}$ is Walrasian if there exists an allocation $(x, \tau)$ such that $(\mathbf{p},(x, \tau))$ is a Walrasian equilibrium.

THEOREM 1: If $p$ is Walrasian then $p=(0,0, \ldots)$.

PROOF: Appendix.

Clearly $\mathbf{p}=(0,0, \ldots)$ and the allocation $x_{h}=(0,1)$ and $\tau_{h}=t$ for each $h \in G_{B}^{t}$ and $x_{h}=(0,0)$ and $\tau_{h}=t$ for each $h \in G_{S}^{t}$ is a Walrasian equilibrium, and so a Walrasian equilibrium exists. (The excess supply of the indivisible good at date 0 is consistent with the date zero indivisible good having a price of zero.) The Walrasian equilibrium is not unique. When the price of the indivisible good is zero at each date, then each seller obtains a utility of zero regardless

\footnotetext{
${ }^{3}$ Our definition of Walrasian equilibrium is similar to the one in Schmidt and Aliprantis [10]. In both definitions there is only a spot market price for each good at each date.
} 
of the date (or even whether or not) it supplies a unit of the indivisible good. Nonetheless, in any Walrasian equilibrium each generation $t$ buyer obtains a unit of date $t$ indivisible good at a price of zero, the utility of each buyer is one, and the utility of each seller is zero.

\section{A Model of an Intermediated Market}

Under the Walrasian trading procedure the allocation of each agent maximizes his utility given his budget. In our model of an intermediated market, each agent's feasible trades are no longer described by his budget set; rather, they are determined by the intermediary's bid and ask price, the bargained price in the private trading market, and the probability that entry in either the mediated or the private trading market concludes with trade. Each of these is endogenously determined in our model of intermediation, just as the budget set is endogenously determined under the Walrasian trading procedure. In the intermediated market each buyer and seller follows an optimal policy of entering the mediated or the private trading market. The policy an agent follows determines a (possibly degenerate) probability distribution over allocations for him.

In our model there is an ocean of buyers and sellers and a single distinguished agent, the intermediary. At each date, each buyer and seller born, but not having yet traded, chooses whether to attempt to trade with the intermediary or whether to attempt to trade privately. Agents entering the mediated market potentially trade immediately with the intermediary at the bid or ask price, but sacrifice the spread as potential gains to trade. The private trading market is modeled as a random matching market. An agent entering the matching market shares all the gains to trade with his partner once matched, but may experience costly delay in being matched. The bid price $P_{b}$ and the ask price $P_{a}$ are chosen by the intermediary to maximize his profits.

An important aspect of the mediated market is that the intermediary is not endowed with an inventory of the traded good, nor can he accumulate one. The intermediary may only cross trades and so, if unequal measures of buyers and sellers enter the mediated market, the intermediary must ration the type of agent entering in greater measure. It is assumed that the intermediary rations only the type of agent entering the mediated market in greater measure and that agents of the same type are rationed with the same probability. Let $m_{i}$ denote the 
measure of agents of type $i \in\{S, B\}$ born but not having yet traded, and let $\lambda_{i}$ denote the proportion of those agents entering the mediated market. The probability that a type $i$ agent trades when entering the mediated market, denoted by $\rho_{i}$, is

$$
\rho_{i}= \begin{cases}\frac{\lambda_{-i} m_{-i}}{\lambda_{i} m_{i}} & \text { if } \lambda_{-i} m_{-i} \leq \lambda_{i} m_{i} \\ 1 & \text { if } \lambda_{-i} m_{-i}>\lambda_{i} m_{i} .\end{cases}
$$

The notation " $-i$ " is used to refer to agents not of type $i$.

Attention is restricted to situations where $P_{a}, P_{b}, m_{i}$, and $\lambda_{i}$ are stationary for each $i \in$ $\{S, B\}$. In a steady state, at each date a measure $\lambda_{i} \rho_{i} m_{i}$ of type $i$ agents trade in the mediated market and then exit. (It is unambiguous to refer to volume as $\lambda_{i} \rho_{i} m_{i}$ since by (1) we have $\left.\lambda_{S} \rho_{S} m_{S}=\lambda_{B} \rho_{B} m_{B}.\right)$ Those agents who enter the mediated market and are rationed, at the next date again choose whether to enter the mediated or the matching market. Since at each date a measure $\nu$ or greater of each type of agent is born, we have $m_{i} \geq \nu$ for each $i \in\{S, B\}$.

When the intermediary crosses a trade, transferring a unit of the indivisible good from a seller to a buyer, the seller receives a price of $P_{b}$ while the buyer pays a price of $P_{a}$. Agents have rational conjectures about their probability of trading when entering the mediated or the matching market. Hence when $R_{B}=1-P_{a}$ and $R_{S}=P_{b}$, the expected reward to a type $i$ agent to entering the mediated market is $\rho_{i} R_{i}$. The difference $1-R_{B}-R_{S}=P_{a}-P_{b}$ is the "spread" and represents the profit to the intermediary from crossing a single trade.

Those agents not entering the mediated market enter the matching market. The probability that a type $i$ agent finds a partner when entering the matching market depends upon the measure of each type of agent entering the matching market. In particular, the probability an agent of type $i$ is matched is

$$
\alpha_{i}=k \frac{\left(1-\lambda_{-i}\right) m_{-i}}{\left(1-\lambda_{i}\right) m_{i}+\left(1-\lambda_{-i}\right) m_{-i}}
$$

where $\left(1-\lambda_{i}\right) m_{i}$ is the measure of agents of type $i$ entering the matching market, and $k \in(0,1]$ is an exogenous parameter indexing the efficiency of the random matching process. ${ }^{4}$ (The same

${ }^{4}$ The matching process has the following interpretation. For agent $h$, let $E$ denote the event that "agent $h$ meets another agent" and let $F$ denote the event that "agent $h$ meets an agent of type $i$." We consider the family of matching processes where for each agent in the matching market $(i) \operatorname{Prob}(E)=k$ and $(i i) \operatorname{Prob}(F \mid E)=$ $\frac{\left(1-\lambda_{i}\right) m_{i}}{\left(1-\lambda_{i}\right) m_{i}+\left(1-\lambda_{-i}\right) m_{-i}}$. Condition $(i)$ says that each agent has the same probability of meeting another agent. (The matching process itself does not differentiate between buyers and sellers.) Condition (ii) says that the probability that an agent meets an agent of type $i$ is equal to the proportion of agents in the matching market that are of 
"matching technology," for $k=1$, is used in Gale [2].) When each match ends with trade, at each date a measure $\alpha_{i}\left(1-\lambda_{i}\right) m_{i}$ of type $i$ agents trade in the matching market and then exit. Those agents who enter the matching market, but who are not matched, again choose whether to enter the mediated or the matching market at the next date.

Since there is an (undiscounted) unit gain to trade in any match, the net surplus of a match is one minus the sum of the buyer's and seller's disagreement payoff. Let $N_{i}$ denote the surplus negotiated by a type $i$ agent when matched. We assume that when the net surplus is nonnegative, each match ends with trade. In this case, $N_{S}+N_{B}=1$ and a matched buyer and seller exchange a unit of the indivisible good at a price of $N_{\mathcal{S}}$. If the net surplus is negative, then bargaining ends with disagreement and $N_{i}$ is defined to be zero for each $i \in\{S, B\}$. The expected reward to an agent of type $i$ to entering the matching market is $\alpha_{i} N_{i}$.

When $\rho_{i}, \alpha_{i}, R_{i}$, and $N_{i}$ are stationary, the problem of choosing an optimal policy for entering the mediated and the matching market is a stationary discounted dynamic programming problem. Agents are assumed to be von Neumann-Morgenstern expected utility maximizers. Let $V_{i}$ denote the expected utility of an agent of type $i$ under the optimal policy. It is well known (see Theorem 2.1 of Ross [6], for example) that $V_{i}$ satisfies the optimality equation ${ }^{5}$

$$
V_{i}=\max \left\{\rho_{i} R_{i}+\left(1-\rho_{i}\right) \delta V_{i}, \alpha_{i} N_{i}+\left(1-\alpha_{i}\right) \delta V_{i}\right\}
$$

The disagreement payoff of a matched agent of type $i$ is $\delta V_{i}$, so the net surplus of a match is $1-\delta V_{S}-\delta V_{B}$. Since each agent is able to obtain a utility of zero by consuming his endowment, attention is restricted to situations where $V_{S} \geq 0$ and $V_{B} \geq 0$.

type $i$. A match is a meeting of two agents of the opposite type.

${ }^{5}$ An agent of type $i$ faces a discounted dynamic programming problem with two states and two actions \{enter mediated, enter matching\}. In state 1 an agent remains in the market while in state 2 an agent has exited the market. In state 1 , the action "enter mediated" yields an expected reward of $\rho_{i} R_{i}$ and the transition probability to state 1 and 2 is $\rho_{i}$ and $1-\rho_{i}$, respectively. The action "enter matching" yields an expected reward of $\alpha_{i} N_{i}$ and the transition probability to state 1 and 2 is $\alpha_{i}$ and $1-\alpha_{i}$, respectively. State 2 is an absorbing state. The expected reward in state 2 is zero for either action. The value equations satisfy

$$
\begin{aligned}
& V_{i}(1)=\max \left\{\rho_{i} R_{i}+\delta\left[\rho_{i} V_{i}(2)+\left(1-\rho_{i}\right) V_{i}(1)\right], \alpha_{i} N_{i}+\delta\left[\alpha_{i} V_{i}(2)+\left(1-\alpha_{i}\right) V_{i}(1)\right]\right\} \\
& V_{i}(2)=0+\delta V_{i}(2)
\end{aligned}
$$

The second of these equations implies that $V_{i}(2)=0$. The first equation then reduces to equation (3) where $V_{i}$ denotes $V_{i}(1)$. 
A matched buyer and seller are assumed to negotiate a price for the indivisible good which evenly splits the net surplus of their match when the net surplus is non-negative. Therefore

$$
N_{i}= \begin{cases}\frac{1-\delta V_{S}-\delta V_{B}}{2}+\delta V_{i} & \text { if } 1-\delta V_{S}-\delta V_{B} \geq 0 \\ 0 & \text { otherwise. }\end{cases}
$$

Since $V_{i} \geq 0$, we have $N_{i} \geq 0$. Using Nash rather than strategic bargaining simplifies the analysis, allowing us to focus on the issues of interest.

The value to an agent of type $i$ of entering the mediated market and following the optimal policy thereafter, is $\rho_{i} R_{i}+\left(1-\rho_{i}\right) \delta V_{i}$. The value of entering the matching market and following the optimal policy thereafter is $\alpha_{i} N_{i}+\left(1-\alpha_{i}\right) \delta V_{i}$. It is well known that there is a stationary policy which is optimal. (See Theorem 2.2 of Ross [6].) In particular, if $\rho_{i} R_{i}+\left(1-\rho_{i}\right) \delta V_{i}$ is greater than $\alpha_{i} N_{i}+\left(1-\alpha_{i}\right) \delta V_{i}$, then the policy of entering the mediated market at each date is optimal. Therefore, the proportion of type $i$ agents entering each market is related to the value of entering each market as follows

$$
\rho_{i} R_{i}+\left(1-\rho_{i}\right) \delta V_{i}\left(\begin{array}{c}
> \\
<
\end{array}\right) \alpha_{i} N_{i}+\left(1-\alpha_{i}\right) \delta V_{i} \Rightarrow \lambda_{i}=\left(\begin{array}{c}
1 \\
0
\end{array}\right) \text {. }
$$

Either policy is optimal when the value of entering each market is equal. One can also interpret $\lambda_{i}$ as, for each agent of type $i$, the probability that he enters the mediated market. Then the interpretation of (5) is that a type $i$ agent enters the market that has the higher value and mixes only if the value of entering each market is the same.

In a steady state, at each date exits from the mediated market plus exits from the matching market are exactly balanced by entry. Thus, for each $i \in\{S, B\}, m_{i}$ is related to $\lambda_{i}, \rho_{i}$, and $\alpha_{i}$ by the equation

$$
\lambda_{i} \rho_{i} m_{i}+\alpha_{i}\left(1-\lambda_{i}\right) m_{i}=\nu
$$

Equation (6) presumes that each match ends with trade, which is indeed the case when entering the matching market is optimal for both types. ${ }^{6}$ Since buyers and sellers exit in pairs from each market, it is natural to require that

$$
m_{S}-m_{B}=\Delta
$$

\footnotetext{
${ }^{6}$ That entering the matching market is optimal for both types implies that $V_{i}=\alpha_{i} N_{i}+\left(1-\alpha_{i}\right) \delta V_{i}$ for each $i \in\{S, B\}$. Moreover, we have that $N_{i}=\frac{1-\delta V_{S}-\delta V_{B}}{2}+\delta V_{i}$ if $1-\delta V_{S}-\delta V_{B} \geq 0$, and $N_{i}=0$ otherwise. This system of equations has a unique solution where $V_{i}=\frac{\alpha_{i}}{2-\delta(2-k)}$ for each $i \in\{S, B\}$. This implies $\delta V_{S}+\delta V_{B}=$ $\frac{\delta k}{2-\delta(2-k)}<1$, and therefore each match ends with trade.
} 
In other words, the steady-state difference between the measure of sellers born, but not having yet traded, and the measure of buyers born, but not having yet traded, must be the same as the difference in the measures at date zero.

A solution to the system of equations (1)-(7) represents a steady state of the intermediated market when each agent follows an optimal policy of entering the mediated and matching market given the bid and ask price, the price negotiated in the private trading market, and the entry policy of every other agent. We refer to such a solution as a quasi-equilibrium. A quasiequilibrium can be viewed as a conjecture by the intermediary of the steady state that prevails for given bid and ask prices. A quasi-equilibrium need not be a full equilibrium as the bid and ask prices need not maximize the intermediary's profit.

Definition. The vector $\left\{R_{i}, V_{i}, m_{i}, \lambda_{i}, \alpha_{i}, \rho_{i}\right\}_{i=S, B}$ is a quasi-equilibrium if it is a solution to the system of equations (1)-(7).

Let $Q$ denote the set of quasi-equilibria. Given a quasi-equilibrium $\left\{R_{i}, V_{i}, m_{i}, \lambda_{i}, \alpha_{i}, \rho_{i}\right\}_{i=S, B}$, the profit to the intermediary is $\left(1-R_{B}-R_{S}\right) \lambda_{i} m_{i} \rho_{i}$. Let $\Pi: Q \rightarrow \Re$ give the profit to the intermediary for each quasi-equilibrium. The intermediary chooses bid and ask prices to maximize its profits, therefore we define an equilibrium with intermediation as follows.

Definition. We say that $q^{*} \in Q$ is an equilibrium with intermediation if for all $q \in Q$ that $\Pi\left(q^{*}\right) \geq \Pi(q)$.

We are interested in intermediation when there is an active private trading market (i.e. a positive proportion of at least one type of agent enters the matching market) and we have implicitly restricted attention to this case. To see this notice that in any quasi-equilibrium we have $\lambda_{i}<1$ for some $i \in\{S, B\}$, and therefore the matching market is active, since otherwise $\alpha_{i}$ is not given by (2). If the matching market were inactive, then an agent entering the matching market does not find a partner. In this case, the intermediary no longer faces any competition from the matching market and he can extract all the gains to trade. The definition of quasiequilibrium rules out this kind of degenerate situation. ${ }^{7}$

\footnotetext{
${ }^{7}$ The definition of quasi-equilibrium rules out, for example, the conjecture by the intermediary that all agents
} 
The definition of quasi-equilibrium also rules out situations where the mediated market is inactive. (If $\lambda_{S}=\lambda_{B}=0$ then $\rho_{i}$ is not given by (1).) If the mediated market is inactive, then an agent entering the mediated market trades with probability zero since the intermediary only crosses trades. This situation is also uninteresting as the intermediary cannot attract entry regardless of the bid and ask prices it sets.

\section{Equilibrium with Intermediation is Walrasian}

The main result of this section is that in every equilibrium with intermediation the bid price is zero and the ask price, which depends on the discount factor, is positive but less than one. As the cost of delay vanishes, the ask price goes to zero, and the intermediary buys and sells the indivisible good at each date at its Walrasian equilibrium price. Our first result is that an equilibrium with intermediation exists.

THEOREM 2: An equilibrium with intermediation exists.

Theorem 3 shows that equilibria with intermediation differ only in the proportion of sellers entering the mediated market and in the probability that a seller entering the mediated market trades.

THEOREM 3: Every equilibrium with intermediation $\left\{R_{i}, V_{i}, m_{i}, \lambda_{i}, \alpha_{i}, \rho_{i}\right\}_{i=S, B}$ satisfies:

(i) $\quad R_{B}=V_{B}=\frac{k}{2-2 \delta+\delta k}, R_{S}=V_{S}=0$;

(ii) $m_{B}=\nu, m_{S}=\nu+\Delta$;

(iii) $\alpha_{B}=k, \alpha_{S}=0$;

(iv) $\lambda_{B}=1, \frac{\nu}{\nu+\Delta} \leq \lambda_{S}<1$;

(v) $\rho_{B}=1, \rho_{S}=\frac{\nu}{\lambda_{S}(\nu+\Delta)}$.

Every equilibrium with intermediation has the following characteristics. At each date, each buyer born, but not having yet traded, enters the mediated market and, since $\rho_{B}=1$, he trades enter the mediated market when the ask price is one and the bid price is zero. 
immediately. Therefore, each generation $t$ buyer purchases one unit of date $t$ indivisible good at the ask price $P_{a}=1-R_{B}=\frac{(1-\delta)(2-k)}{2-2 \delta+\delta k}$, which is greater than zero but less than one. At each date, each seller born, but not having yet traded, enters the mediated market with probability $\lambda_{S}$. Since $\rho_{S}>0$ and $\alpha_{S}=0$, each generation $t$ seller eventually supplies one unit of the indivisible good at the bid price $P_{b}=R_{S}=0.8$ (Sellers may be rationed in equilibrium since $\rho_{S}<1$ when $\lambda_{S}>\frac{\nu}{\nu+\Delta}$.) All trade takes place in the mediated market. Hence the volume of trade in the mediated market is $\nu$, the maximum possible steady-state volume. The spread is $1-R_{B}-R_{S}=\frac{(1-\delta)(2-k)}{2-2 \delta+\delta k}$, and therefore the intermediary's profit in equilibrium is $\frac{(1-\delta)(2-k)}{2-2 \delta+\delta k} \nu$. Since the bid price is zero, the intermediary and buyers capture all of the gains to trade.

The comparative statics with respect to the efficiency of the matching process go in the expected direction: As $k$ increases, the random matching process thereby becoming more efficient, the intermediary's spread (and hence his profit since volume does not depend on $k$ ) both decrease.

Of primary interest is the equilibrium behavior of the intermediary as the cost of delay vanishes. As the discount factor approaches one, the ask price obtained in the limit is zero since $\lim _{\delta \rightarrow 1} \frac{(1-\delta)(2-k)}{2-2 \delta+\delta k}=0$. The spread also goes to zero as the cost of delay vanishes, since the bid price is zero regardless of the cost of delay. In the limit, the intermediary buys and sells the indivisible good at each date at its Walrasian equilibrium price of zero. Each buyer and seller obtains his Walrasian equilibrium payoff.

In the remainder of this section, we prove Theorems 2 and 3 and we discuss the intuition underlying the result that when the costs of delay are small, the intermediary sets the bid and ask price of the indivisible good nearly equal to its Walrasian equilibrium price. Lemma 1 establishes some properties of quasi-equilibria. The properties established are also properties of equilibria with intermediation since an equilibrium with intermediation is also a quasi-equilibrium. The proof of Lemma 1 is given in the appendix.

LEMMA 1: If $\left\{R_{i}, V_{i}, m_{i}, \lambda_{i}, \alpha_{i}, \rho_{i}\right\}_{i=S, B} \in Q$ then (i) $\lambda_{i}>0$ for each $i \in\{S, B\}$, (ii) $V_{S}+V_{B} \geq$ $\frac{k}{2-2 \delta+\delta k}$, and (iii) $R_{i}=V_{i} \frac{1-\delta\left(1-\rho_{i}\right)}{\rho_{i}}$ for each $i \in\{S, B\}$.

\footnotetext{
${ }^{8}$ Here we interpret $\lambda_{S}$ as the probability that a seller enters the mediated market. A seller entering the matching market does not trade since $\alpha_{S}=0$. Since $\lambda_{S}>0$ and $\rho_{S}>0$, each seller eventually trades one unit of the indivisible good in the mediated market with probability one.
} 
Part $(i)$ of Lemma 1 shows that a positive proportion of each type of agent enters the mediated market in each quasi-equilibrium. This result holds since in a quasi-equilibrium at least one type of agent enters the mediated market, but it cannot be optimal for only one type to enter the mediated market. An implication of Part ( $i)$ of Lemma 1 is that volume is positive in each quasi-equilibrium. Part ( $i)$ does not imply that volume is positive for every bid and ask price, since not every combination of bid and ask prices can be realized as part of a solution to (1)-(7).

Part ( $i i)$ of Lemma 1 shows that the sum $V_{S}+V_{B}$ is at least as great as $\frac{k}{2-2 \delta+\delta k}$. This result holds since in a quasi-equilibrium each agent has the opportunity to trade in an active private trading market.

Part (iii) of the Lemma holds since to induce a type $i$ agent to enter the mediated market the intermediary must offer him a share of the surplus $R_{i}$ at least as great as $V_{i}$. Together, parts (ii) and (iii) of the Lemma imply that in a quasi-equilibrium the spread is no greater than $1-\frac{k}{2-2 \delta+\delta k}$. Moreover, if $V_{i}$ is positive and $0<\rho_{i}<1$ for some type $i \in\{S, B\}$, then $R_{i}$ must be greater than $V_{i}$ and the spread is strictly less than $1-\frac{k}{2-2 \delta+\delta k}$.

We are now prepared to prove the main results of this section.

PROOF OF THEOREM 2: We show that the vector $q^{*}=\left\{R_{i}^{*}, V_{i}^{*}, m_{i}^{*}, \lambda_{i}^{*}, \alpha_{i}^{*}, \rho_{i}^{*}\right\}_{i=S, B}$ given by

$$
R_{B}^{*}=\frac{k}{2-2 \delta+\delta k}, \quad V_{B}^{*}=\frac{k}{2-2 \delta+\delta k}, \quad m_{B}^{*}=\nu, \quad \lambda_{B}^{*}=1, \quad \alpha_{B}^{*}=k, \quad \rho_{B}^{*}=1,
$$

and

$$
R_{S}^{*}=0, \quad V_{S}^{*}=0, \quad m_{S}^{*}=\nu+\Delta, \quad \lambda_{S}^{*}=\frac{\nu}{\nu+\Delta}, \quad \alpha_{S}^{*}=0, \quad \rho_{S}^{*}=1,
$$

is an equilibrium with intermediation. It is easy to verify that $q^{*} \in Q$. We then need to show that for each $q=\left\{R_{i}, V_{i}, m_{i}, \lambda_{i}, \alpha_{i}, \rho_{i}\right\}_{i=S, B} \in Q$ one has

$$
\Pi(q)=\left(1-R_{B}-R_{S}\right) \lambda_{i} m_{i} \rho_{i} \leq \frac{(1-\delta)(2-k)}{2-2 \delta+\delta k} \nu=\Pi\left(q^{*}\right) .
$$

Part ( $i)$ of Lemma 1 and equation (1) yield $\rho_{i}>0$ for each $i \in\{S, B\}$. Therefore $\frac{1-\delta\left(1-\rho_{i}\right)}{\rho_{i}}$ is well defined, and Part (iii) of Lemma 1 and $V_{i} \geq 0$ imply $R_{i} \geq V_{i}$ for each $i \in\{S, B\}$. Part (ii) of Lemma 1 and $R_{i} \geq V_{i}$ for each $i \in\{S, B\}$ imply that $1-R_{B}-R_{S} \leq \frac{(1-\delta)(2-k)}{2-2 \delta+\delta k}$. The inequality is obtained by noting that $\lambda_{i} \rho_{i} m_{i} \leq \nu$ by $(6)$. 
The proof of Theorem 3 follows.

PROOF OF THEOREM 3: Suppose that $q=\left\{R_{i}, V_{i}, m_{i}, \lambda_{i}, \alpha_{i}, \rho_{i}\right\}_{i=S, B}$ is an equilibrium with intermediation. We show that $\lambda_{i} \rho_{i} m_{i}=\nu$ and $R_{B}+R_{S}=\frac{k}{2-2 \delta+\delta k}$. By (6) we have that $\lambda_{i} \rho_{i} m_{i} \leq \nu$ and by Part (ii) and (iii) of Lemma 1 we have that $R_{B}+R_{S} \geq \frac{k}{2-2 \delta+\delta k}$. If either $\lambda_{i} \rho_{i} m_{i}<\nu$ or $R_{B}+R_{S}>\frac{k}{2-2 \delta+\delta k}$, then

$$
\Pi(q)=\left(1-R_{B}-R_{S}\right) \lambda_{i} \rho_{i} m_{i}<\left(1-\frac{k}{2-2 \delta+\delta k}\right) \nu=\frac{(1-\delta)(2-k)}{2-2 \delta+\delta k} \nu=\Pi\left(q^{*}\right),
$$

contradicting that $q$ is an equilibrium with intermediation.

We show that $R_{B}+R_{S}=\frac{k}{2-2 \delta+\delta k}$ and Lemma 1 imply that:

(a) $V_{S}+V_{B}=\frac{k}{2-2 \delta+\delta k}$.

(b) For each $i \in\{S, B\}: V_{i}>0$ implies $\rho_{i}=1$.

Since $R_{B}+R_{S}=\frac{k}{2-2 \delta+\delta k}$ and since $R_{i} \geq V_{i}$ for each $i \in\{S, B\}$ by Part (iii) of Lemma 1, then (a) holds by Part (ii) of Lemma 1. Suppose contrary to (b) that $V_{i}>0$ and $\rho_{i}<1$. Then $R_{i}>V_{i}$ by Part (iii) of Lemma 1 . Since $R_{i}>V_{i}$ and $R_{-i} \geq V_{-i}$, then $R_{S}+R_{B}>V_{S}+V_{B}=\frac{k}{2-2 \delta+\delta k}$ which is a contradiction.

We now show that $\lambda_{B}=1$. Since $\lambda_{i} \rho_{i} m_{i}=\nu$ we have by (6) that $\alpha_{i}\left(1-\lambda_{i}\right) m_{i}=0$. Therefore either $\lambda_{i}=1$ or $\alpha_{i}=0$. But $\alpha_{i}$ is zero only if $\lambda_{-i}=1$. Therefore, either $\lambda_{i}=1$ or $\lambda_{-i}=1$. Suppose that $\lambda_{B}<1$. Then $\lambda_{S}=1$. Moreover, $m_{S}=m_{B}+\Delta>\nu$ and $\lambda_{S} \rho_{S} m_{S}=\nu$ imply that $\rho_{S}<1$. Observation (a) yields $1-\delta V_{S}-\delta V_{B}>0$, and therefore by (4) we have that $N_{S}=\frac{1-\delta V_{S}-\delta V_{B}}{2}+\delta V_{S}>0$. Since $\lambda_{B}<1$, it follows from (2) that $\alpha_{S}>0$. By (3), we have

$$
V_{S} \geq \alpha_{S} N_{S}+\left(1-\alpha_{S}\right) \delta V_{S}
$$

Thus $N_{S}>0$ and $\alpha_{S}>0$, and therefore $V_{S}>0$, which contradicts (b) since $\rho_{S}<1$.

We now show that $V_{S}=0$ and $V_{B}=\frac{k}{2-2 \delta+\delta k}$. In a quasi-equilibrium either $\lambda_{S}<1$ or $\lambda_{B}<1$. (If both $\lambda_{S}=\lambda_{B}=1$, then the matching market is inactive and $\alpha_{i}$ is not given by (2).) Therefore, $\lambda_{B}=1$ implies that $\lambda_{S}<1$. Moreover, $\lambda_{B}=1$ implies by (2) that $\alpha_{S}=0$. Since $\lambda_{S} \neq 1$, then (3) and (5) imply that $V_{S}=\alpha_{S} N_{S}+\left(1-\alpha_{S}\right) \delta V_{S}$. As $\alpha_{S}=0$ and $\delta<1$, we have $V_{S}=0$. Part (iii) of Lemma 1 implies that $R_{S}=0$. From $(a)$ it follows that $V_{B}=\frac{k}{2-2 \delta+\delta k}$. 
From $V_{B}>0$ and $(b)$, we have $\rho_{B}=1$. Part (iii) of Lemma 1 and $V_{B}=\frac{k}{2-2 \delta+\delta k}$ then imply that $R_{B}=\frac{k}{2-2 \delta+\delta k}$.

It is only left to be shown that $m_{S}=\nu+\Delta, m_{B}=\nu, \lambda_{S} \geq \frac{\nu}{\nu+\Delta}$, and $\rho_{S}=\frac{\nu}{\lambda_{S}(\nu+\Delta)}$. Since $\rho_{B}=1$, by (1) we have $\lambda_{S} m_{S} \geq \lambda_{B} m_{B}$. Moreover, $\lambda_{B} \rho_{B} m_{B}=\nu$ and $\lambda_{B}=\rho_{B}=1$ imply that $m_{B}=\nu$, and therefore $m_{S}=\nu+\Delta$ by (7). Then $\lambda_{S} m_{S} \geq \lambda_{B} m_{B}$ implies that $\lambda_{S} \geq \frac{\nu}{\nu+\Delta}$, and $\lambda_{S} \rho_{S} m_{S}=\nu$ implies that $\rho_{S}=\frac{\nu}{\lambda_{S}(\nu+\Delta)}$.

To understand the result that the intermediary sets nearly Walrasian bid and ask prices when the cost of delay is small, it is useful to note that there are quasi-equilibria in which the intermediary and sellers capture all of the gains to trade. Consider, for example, the quasiequilibrium where $V_{S}=\frac{k}{2-2 \delta+\delta k}, V_{B}=0, m_{S}=2 \nu+\Delta, m_{B}=2 \nu, \lambda_{S}=1, \lambda_{B}=\frac{1}{2}, \alpha_{S}=k, \alpha_{B}=$ $0, \rho_{S}=\frac{\nu}{2 \nu+\Delta}, \rho_{B}=0, R_{S}=V_{S} \frac{1-\delta\left(1-\rho_{S}\right)}{\rho_{S}}$, and $R_{B}=0$. In this quasi-equilibrium the bid price is $\frac{k}{2-2 \delta+\delta k} \frac{1-\delta\left(1-\rho_{S}\right)}{\rho_{S}}$ and the ask price is 1 . As the cost of delay vanishes, in the limit the bid and the ask price are both one. This quasi-equilibrium is not an equilibrium with intermediation, however, as these bid and ask prices do not maximize the intermediary's profit. Although the volume of trade in the mediated market is $\nu$, sellers have a positive value and are rationed, and therefore the spread is less than $1-\frac{k}{2-2 \delta+\delta k}$.

In the next section we show that when agents only have the opportunity to trade privately, then the price of the indivisible good at each date is positive even as the cost of delay vanishes. We conclude that some degree of centralized trade, like that provided by an intermediary, is necessary for the market outcome to be Walrasian.

\section{Equilibrium with Only Private Trading}

We now consider the trading procedure obtained by removing the possibility of trading through the intermediary from the model of intermediation. In this case, all trade is private and at each date the price of the indivisible good is determined by bargaining between matched buyers and sellers. We define an equilibrium with private trading to represent equilibrium in the market when trade is completely decentralized. 
Definition: The vector $\left\{V_{i}, m_{i}, \alpha_{i},\right\}_{i=S, B}$ is an equilibrium with private trading if for each $i \in$ $\{S, B\}$ :

(i) $\quad \alpha_{i}=k \frac{m_{-i}}{m_{i}+m_{-i}} ;$

(ii) $V_{i}=\alpha_{i}\left(\frac{1-\delta V_{S}-\delta V_{B}}{2}+\delta V_{i}\right)+\left(1-\alpha_{i}\right) \delta V_{i}$

(iii) $\alpha_{i} m_{i}=\nu$;

(iv) $m_{S}-m_{B}=\Delta$.

Equation (i) defines matching probabilities when there is only private trade. Equation (ii) gives the value to an agent of type $i$ (when unmatched, prior to the random matching of agents) when the price of the indivisible good in each match is determined by Nash bargaining. Equation (iii) says that in a steady state, exits are balanced by entry. Equation (iv) requires that the difference at date zero between the measure of sellers born but not having yet traded and the measure of buyers born but not having yet traded is preserved in a steady state.

It turns out that there is a unique equilibrium with private trading, denoted by the vector $\left\{V_{i}^{*}, m_{i}^{*}, \alpha_{i}^{*}\right\}_{i=S, B}$. In this equilibrium

$$
V_{i}^{*}=\frac{\alpha_{i}^{*}}{2-\delta(2-k)}>0
$$

where $0<\alpha_{i}^{*}<k .^{9}$ By equation (2) we have that $\alpha_{S}^{*}+\alpha_{B}^{*}=k$, and therefore

$$
\dot{V}_{S}^{*}+V_{B}^{*}=\frac{\alpha_{S}^{*}+\alpha_{B}^{*}}{2-\delta(2-k)}=\frac{k}{2-\delta(2-k)} \text {. }
$$

The sum is less than one since, when all trade is private, each agent typically trades only following costly delay. The price of the indivisible good at each date is given by $\frac{1-\delta V_{S}^{*}-\delta V_{\dot{B}}^{*}}{2}+\delta V_{S}^{*}$ and equals $\frac{1-\delta\left(1-\alpha_{S}^{*}\right)}{2-\delta(2-k)}>0$.

When delay costs are positive, each agent in a match has market power and one should not expect the bargained price to be Walrasian. More surprisingly, as the cost of delay vanishes the price of the indivisible good at each date is given by $\lim _{\delta \rightarrow 1} \frac{1-\delta\left(1-\alpha_{s}^{*}\right)}{2-\delta(2-k)}=\frac{\alpha_{s}^{*}}{k}$, which is greater than its Walrasian equilibrium price of zero. Neither buyers nor sellers obtain their Walrasian equilibrium payoff. Buyers obtain $\lim _{\delta \rightarrow 1} \frac{\alpha_{B}^{*}}{2-\delta(2-k)}=\frac{\alpha_{B}^{*}}{k}<1$ and sellers obtain $\frac{\alpha_{S}^{*}}{k}>0$.

This non-Walrasian result does not depend on the assumption that prices are determined by Nash bargaining. Rubinstein and Wolinsky [8] embed the alternating offer game of Rubinstein

\footnotetext{
${ }^{9}$ The remainder of the equilibrium vector is $m_{B}^{*}=\frac{2 \gamma-\Delta+\left(4 \gamma^{2}+\Delta^{2}\right)^{2 / 2}}{2}$ and $m_{S}^{*}=\frac{2 \gamma+\Delta+\left(4 \gamma^{2}+\Delta^{2}\right)^{2 / 2}}{2}$, where $\gamma=\frac{\nu}{k}$ and $\alpha_{i}^{*}=k \frac{m_{-i}^{*}}{m_{-i}^{*}+m_{i}^{*}}>0$.
} 
[7] into a model where there is only private trade. They show that even with strategic bargaining, the market outcome is not close to the Walrasian outcome as the cost of delay vanishes. ${ }^{10}$

\section{Concluding Remarks}

It is natural to ask how the entry of an intermediary into a market where heretofore all trade was private affects the welfare of buyers and sellers. It turns out that the average welfare of buyers and sellers, as measured by $V_{S}+V_{B}$, is the same in the equilibrium with private trading as in the equilibrium with intermediation. The effect of the entry of an intermediary, and therefore the introduction of the possibility of centralized trade, is to shift the capture of the gains to trade towards the type of agent present in the market in smaller measure.

In the equilibrium with intermediation there is no costly delay; each buyer trades at the date he is born and, since the bid price is zero, each seller obtains a utility of zero regardless of the delay (if any) he experiences prior to trading. Since the average welfare of buyers and sellers is the same in the equilibrium with private trading and in the equilibrium with intermediation, we see that the intermediary captures all the gains arising from the elimination of costly delay. This result is not surprising given that the intermediary is a monopolist.

Other authors have studied models of "large" intermediaries that differ from our model in significant respects. In Yavas [12] and in Gehrig [3] the search market operates for only one period. Moresi [5] characterizes the steady state that prevails in the search market for given bid and ask prices, but does not determine the intermediary's profit maximizing bid and ask. In the present paper the search market operates perpetually and the intermediary sets bid and ask prices to maximize his profits.

\section{Appendix}

Before proving Theorem 1, we state two claims which are used to prove Theorem 1. Claim A is that in a Walrasian equilibrium price sequence, the price of the indivisible good is bounded

\footnotetext{
${ }^{10}$ They obtain this result for a economy where each agent who trades is replaced by an agent of the same type. Their assumption that entry is through replacement, rather than exogenously given as in the present paper, is not essential for their result.
} 
below by zero and above by one. Claim B gives a lower bound on the rate of growth of prices along an arbitrarily long subsequence of dates if $p_{t}>0$ for some $t$. These results are then used to prove Theorem 1 , that in any Walrasian equilibrium $p=(0,0, \ldots)$.

CLAIM A: Let $\mathbf{p}$ be a Walrasian price sequence. Then $0 \leq p_{t} \leq 1$ for each $t$.

PROOF: Suppose that $\mathbf{p}$ is Walrasian. We first show that $p_{t} \leq 1$ for each $t$. Suppose to the contrary that we have $p_{t}>1$ for some $t$. Then $x_{h}^{1}=0$ for each seller $h \in G_{S}^{t}$ since the allocation $\left(p_{t}, 0, t\right)$ is feasible and yields a utility of $p_{t}>0$, while any allocation with $x_{h}^{1}=1$ yields at most a utility of zero (by the budget constraint of seller $h$ ). Thus, there is a date $k \geq t$ such that $\tau_{h}=k$ and $x_{h}^{1}=0$ for a positive measure of sellers $h \in G_{S}^{t}$. Utility maximization and $\tau_{h}=k$ imply that $p_{k} \delta^{k-t} \geq p_{t}$, and therefore $p_{k}>1$. By Walras's law, since $p_{k}>0$ the market-clearing condition for date $k$ indivisible good must hold as an equality. ${ }^{11}$ Therefore, we must have $\tau_{h}=k$ and $x_{h}^{1}=1$ for a positive measure of buyers $h \in G_{B}^{0} \cup \ldots \cup G_{B}^{k}$. But the allocation $\left(-p_{k}, 1, k\right)$ yields a utility of less than zero for any such buyer, while the allocation $(0,0, k)$ is feasible and yields a utility of zero. Therefore, utility maximization is contradicted. The symmetric argument shows that $p_{t} \geq 0$ for each $t$.

CLAIM B: Let $\mathbf{p}$ be a Walrasian price sequence. If $p_{t}>0$, then for any $n \geq t$ there exists an $m>n$, such that $p_{t} \leq p_{m} \delta^{m-t}$.

PROOF: Let $(p,(x, \tau))$ be a Walrasian equilibrium and suppose that $p_{t}>0$ for some $t$. We first show that $p_{t}>0$ implies that

$$
\int_{\left\{h \in G \mid \tau_{h} \leq t\right\}}\left(x_{h}^{1}-\omega_{h}^{1}\right) d h=0 .
$$

We begin by showing that the market-clearing condition for the indivisible good at each date holds with equality for each date $k \leq t$. By Claim A, we have $p_{k} \geq 0$. If $p_{k}>0$ then Walras's Law implies that $\int_{\left\{h \in G \mid \tau_{h}=k\right\}}\left(x_{h}^{1}-\omega_{h}^{1}\right) d h=0$. If $p_{k}=0$ and $k \leq t$, then utility maximization implies that $\tau_{h} \neq k$ for each $h \in G_{S}^{0} \cup \ldots \cup G_{S}^{k}$, since seller $h$ obtains at most a utility of

\footnotetext{
${ }^{11}$ In this economy, for each $k$ Walras's Law states
}

$$
\int_{\left\{h \in G \mid \tau_{h}=k\right\}}\left(x_{h}^{0}-\omega_{h}^{0}\right) d h+p_{k} \int_{\left\{h \in G \mid \tau_{h}=k\right\}}\left(x_{h}^{1}-\omega_{h}^{1}\right) d h=0 .
$$


zero trading at date $k$, while the allocation $\left(p_{t}, 0, t\right)$ is feasible and yields a positive utility. Thus, for each $k \leq t$ we have that $\int_{\left\{h \in G_{S} \mid \tau_{h}=k\right\}}\left(x_{h}^{1}-\omega_{h}^{1}\right) d h=0$. Now $\omega_{h}^{1}=0$ for each buyer $h \in G_{B}^{0} \cup \ldots \cup G_{B}^{k}$, which implies that $\int_{\left\{h \in G_{B} \mid \tau_{h}=k\right\}}\left(x_{h}^{1}-\omega_{h}^{1}\right) d h \geq 0$. Summing these last two integrals yields $\int_{\left\{h \in G \mid \tau_{h}=k\right\}}\left(x_{h}^{1}-\omega_{h}^{1}\right) d h \geq 0$, which together with the market-clearing condition for good 1 at date $k$, implies that $\int_{\left\{h \in G \mid \tau_{h}=k\right\}}\left(x_{h}^{1}-\omega_{h}^{1}\right) d h=0$. Summing over $k \leq t$ we obtain (8).

The Claim is proved by induction. Let $P(n)$ be the proposition "If $p_{t}>0$, then there exists an $m>n$ such that $p_{t} \leq p_{m} \delta^{m-t}$." To prove the Claim we need to establish that $P(n)$ is true for each $n \geq t$. We begin by showing that $P(n)$ is true for $n=t$.

Utility maximization and $p_{t}>0$ implies that for each seller $h \in G_{S}^{k}$, where $k \leq t$, we have $x_{h}^{1}=0$. This follows since the allocation $\left(p_{t}, 0, t\right)$ is feasible and it yields a utility of $\delta^{t-k} p_{t}>0$. In contrast, any allocation with $x_{h}^{1}=1$ must be such that $x_{h}^{0} \leq 0$, and therefore yields at most a utility of zero. Suppose contrary to $P(t)$ that for all $m>t$ we have $p_{t}>p_{m} \delta^{m-t}$. Then $p_{t} \delta^{t-k}>p_{m} \delta^{m-k}$ for all $m>t$, and therefore each generation $k$ seller, $k \leq t$, obtains a higher utility trading at date $t$ than trading at any other date $m>t$. Hence, for each seller $h \in G_{S}^{0} \cup \ldots \cup G_{S}^{t}$ we have $\tau_{h} \leq t, x_{h}^{1}=0$, and $w_{h}^{1}=1$. Thus,

$$
\int_{\left\{h \in G_{S} \mid \tau_{h} \leq t\right\}}\left(x_{h}^{1}-\omega_{h}^{1}\right) d h=-\left[\mu\left(G_{S}^{0}\right)+\ldots+\mu\left(G_{S}^{t}\right)\right] .
$$

Since $x_{h}^{1} \leq 1$ and $\omega_{h}^{1}=0$ for each $h \in G_{B}^{0} \cup \ldots \cup G_{B}^{t}$ we have that $\int_{\left\{h \in G_{B} \mid \tau_{h} \leq t\right\}}\left(x_{h}^{1}-\omega_{h}^{1}\right) d h \leq$ $\mu\left(G_{B}^{0}\right)+\ldots+\mu\left(G_{B}^{t}\right)$. Summing these last two integrals yields

$$
\int_{\left\{h \in G \mid \tau_{h} \leq t\right\}}\left(x_{h}^{1}-\omega_{h}^{1}\right) d h \leq \mu\left(G_{B}^{0}\right)+\ldots+\mu\left(G_{B}^{t}\right)-\left[\mu\left(G_{S}^{0}\right)+\ldots+\mu\left(G_{S}^{t}\right)\right]<0
$$

which contradicts equation (8).

Let $i \geq t$ be arbitrary. We assume that $P(i)$ is true and we show that $P(i+1)$ is true. Since $P(i)$ is true there exists an $m>i$ such that $p_{t} \leq p_{m} \delta^{m-t}$. Suppose $P(i+1)$ is false; i.e. for all $s>i+1$ we have $p_{t}>p_{s} \delta^{s-t}$. Then for all $s>i+1$ we have

$$
p_{m} \delta^{m-t} \geq p_{t}>p_{s} \delta^{s-t}
$$

Clearly, it must be the case that $m=i+1$. (If $m>i+1$ then equation (10) does not hold for $s=m$, which is a contradiction.) 
Since $p_{t}>0$, then (10) implies $p_{i+1}>0$. Utility maximization and $p_{i+1}>0$ imply that for each seller $h \in G_{S}^{0} \cup \ldots \cup G_{S}^{i+1}$ we have $x_{h}^{1}=0$. Now (10) also implies that $p_{i+1} \delta^{i+1-k}>p_{s} \delta^{s-k}$ for all $s>i+1$, and therefore each generation $k$ seller, $k \leq i+1$, obtains a higher utility trading at date $i+1$ than trading at any subsequent date. We have shown for each seller $h \in G_{S}^{0} \cup \ldots \cup G_{S}^{i+1}$ that $\tau_{h} \leq i+1, x_{h}^{1}=0$, and $\omega_{h}^{1}=1$. Therefore

$$
\int_{\left\{h \in G_{S} \mid \tau_{h} \leq i+1\right\}}\left(x_{h}^{1}-\omega_{h}^{1}\right) d h=-\left[\mu\left(G_{S}^{0}\right)+\ldots+\mu\left(G_{S}^{i+1}\right)\right] .
$$

Since $x_{h}^{1} \leq 1$ and $\omega_{h}^{1}=0$ for each $h \in G_{B}^{0} \cup \ldots \cup G_{B}^{i+1}$ we have that $\int_{\left\{h \in G_{B} \mid \tau_{h} \leq i+1\right\}}\left(x_{h}^{1}-\omega_{h}^{1}\right) d h \leq$ $\mu\left(G_{B}^{0}\right)+\ldots+\mu\left(G_{B}^{i+1}\right)$. Therefore,

$$
\int_{\left\{h \in G \mid \tau_{h} \leq i+1\right\}}\left(x_{h}^{1}-\omega_{h}^{1}\right) d h \leq \mu\left(G_{B}^{0}\right)+\ldots+\mu\left(G_{B}^{i+1}\right)-\left[\mu\left(G_{S}^{0}\right)+\ldots+\mu\left(G_{S}^{i+1}\right)\right]<0,
$$

which since $p_{i+1}>0$ contradicts equation (8).

PROOF OF THEOREM 1: Suppose contrary to the theorem that $\mathbf{p} \neq(0,0, \ldots)$. Since by Claim A we have $p_{t} \geq 0$ for all $t$, there is a $t$ such that $p_{t}>0$. By Claim B we have that for any positive integer $n \geq t$, there exists an $m>n$ such that $p_{t} \leq p_{m} \delta^{m-t}$. Since $\delta<1$ this implies that for $n$ sufficiently large there exists an $m$ such that $p_{m}>1$, contradicting Claim A. This contradiction establishes Theorem 1.

The proof of Lemma 1 follows.

PROOF OF LEMMA 1: Proof OF PART ( $i$ ). In a quasi-equilibrium $\lambda_{i}>0$ for some $i \in\{S, B\}$. (If $\lambda_{S}=\lambda_{B}=0$, then $\rho_{i}$ is not given by equation (1).) We show that in fact $\lambda_{i}>0$, for each $i \in\{S, B\}$. Suppose to the contrary that $\lambda_{i}=0$ for some $i \in\{S, B\}$. We show this implies that $V_{-i}=0$. As $\lambda_{i}=0$ and $\lambda_{-i}>0$ we have $\rho_{-i}=0$ by (1) and $\alpha_{-i}>0$ by (2). Since $\lambda_{-i} \neq 0$ then

$$
V_{-i}=\rho_{-i} R_{-i}+\left(1-\rho_{-i}\right) \delta V_{-i} \geq \alpha_{-i} N_{-i}+\left(1-\alpha_{-i}\right) \delta V_{-i},
$$

where the inequality follows from (5) and the equality follows from (3). The equality in (12), $\rho_{-i}=0$, and $\delta<1$, imply that $V_{-i}=0$. The inequality in (12) and $\alpha_{-i}>0$ imply that $N_{-i}=0$. In order to prove Part (i) of the Lemma we consider two cases. 
CASE I: Suppose that $1-\delta V_{S}-\delta V_{B} \geq 0$. In this case we have $N_{-i}=\frac{1-\delta V_{S}-\delta V_{B}}{2}+\delta V_{-i}$ by (4). Since $N_{-i}=0$ and $V_{-i}=0$ we have that $1-\delta V_{S}-\delta V_{B}=0$. Therefore, we also have that $V_{i}=\frac{1}{\delta}$. As $\lambda_{i} \neq 1$, then $(3)$ and $(5)$ yield

$$
V_{i}=\alpha_{i} N_{i}+\left(1-\alpha_{i}\right) \delta V_{i}
$$

Because $V_{i}=\frac{1}{\delta}, 1-\delta V_{S}-\delta V_{B}=0$, and $N_{i}=\frac{1-\delta V_{S}-\delta V_{B}}{2}+\delta V_{i}$, we have $N_{i}=1$. Replacing $N_{i}$ with 1 and $V_{i}$ with $\frac{1}{\delta}$ in (13) gives $\frac{1}{\delta}=1$, which is a contradiction.

CASE II: Suppose that $1-\delta V_{S}-\delta V_{B}<0$. In this case we have $N_{S}=N_{B}=0$ by (4). Equation (13) then implies that $V_{i}=0$. But $V_{i}=0$ and $V_{-i}=0$ (from above) contradicts that $1-\delta V_{S}-\delta V_{B}<0$.

Proof of PART (ii). By equation (5), for each $i \in\{S, B\}$ we have

$$
V_{i} \geq \alpha_{i} N_{i}+\left(1-\alpha_{i}\right) \delta V_{i}
$$

Suppose that $1-\delta V_{S}-\delta V_{B} \geq 0$. Then summing (14) over $i \in\{S, B\}$ and using (4), we have

$$
V_{S}+V_{B} \geq\left(\alpha_{S}+\alpha_{B}\right)\left(\frac{1-\delta V_{S}-\delta V_{B}}{2}\right)+\delta\left(V_{S}+V_{B}\right) \text {. }
$$

Equation (2) implies that $\alpha_{S}+\alpha_{B}=k$. Substituting $\alpha_{S}+\alpha_{B}=k$ into the equation above and rearranging the result yields the desired inequality. If $1-\delta V_{S}-\delta V_{B}<0$, then we have that $V_{S}+V_{B}>\frac{1}{\delta}$. Therefore $\frac{k}{2-2 \delta+\delta k}<1<V_{S}+V_{B}$ and the result is proven.

Proof OF PART (iii). By Lemma 1 , we have $\lambda_{i}>0$ for each $i \in\{S, B\}$. Since $\lambda_{i} \neq 0$ for each $i \in\{S, B\}$, then (3) and (5) imply that $V_{i}=\rho_{i} R_{i}+\left(1-\rho_{i}\right) \delta V_{i}$ for each $i \in\{S, B\}$.

\section{References}

[1] Diamond, P. (1982): "Wage Determination and Efficiency in Search Equilibrium," Review of Economic Studies, 49, 217-227.

[2] Gale, D. (1987): "Limit Theorems for Markets with Sequential Bargaining," Journal of Economic Theory, 43, 20-54. 
[3] Gehrig, T. (1993): "Intermediation in Search Markets," Journal of Economics and Management Strategy, 2, 97-120.

[4] Hahn, F. (1987): "Auctioneer," in The New Palgrave, John Eatwell, Murray Milgate, and Peter Newman, ed., MacMillan Press Limited, New York.

[5] Moresi, S. (1990): "Intermediation in Markets with Sequential Bargaining and Heterogeneous Buyers and Sellers," mimeo.

[6] Ross, S. (1983): Introduction to Stochastic Dynamic Programming, Academic Press, New York.

[7] Rubinstein, Ariel. (1982): "Perfect Equilibrium in a Bargaining Model," Econometrica, 50, 97-110.

[8] Rubinstein, A. and A. Wolinsky. (1985): "Equilibrium in a Market with Sequential Bargaining," Econometrica, 53, 1133-1150.

[9] — and —. (1987): "Middlemen," Quarterly Journal of Economics, 102, 581-593.

[10] Schmidt, D. and C. Aliprantis. (1993): "Price Dynamics in Overlapping Generations Environments," Economic Theory, 3, 541-563.

[11] Wooders, J. (1992): "Markets Without an Auctioneer," Ph.D. dissertation, Cornell University, Ithaca N.Y.

[12] Yavas, A. (1994): "Middlemen in Bilateral Search Markets," Journal of Labor Economics, 12, 406-429. 